Pacific Journal of Mathematics

A CHARACTERIZATION OF THE SUBGROUPS OF THE 


\title{
A CHARACTERIZATION OF THE SUBGROUPS OF THE ADDITIVE RATIONALS
}

\author{
Ross A. Beaumont and H. S. Zuckerman
}

1. Introduction. In the class of abelian groups every element of which (except the identity) has infinite order, the subgroups of the additive group of rational numbers have the simplest structure. These rational groups are the groups of rank one, or generalized cyclic groups, an abelian group $G$ being said to have rank one if for any pair of elements, $a \neq 0, b \neq 0$, in $G$, there exist integers $m, n$, such that $m a=n b \neq 0$. Although many of the properties of these groups are known [1], it seems worthwhile to give a simple characterization from which their properties can easily be derived. This characterization is given in Theorems 1 and 2 of $\S 2$, and the properties of the rational groups are obtained as corollaries of these theorems in $\$ 3$. In $\$ 4$, all rings which have a rational group as additive group are determined.

Let $p_{1}, p_{2}, \cdots, p_{j}, \cdots$ be an enumeration of the primes in their natural order; and associate with each $p_{j}$ an exponent $k_{j}$, where $k_{j}$ is a nonnegative integer or the symbol $\infty$. We consider sequences $i ; k_{1}, k_{2}, \cdots, k_{j}, \cdots$, where $i$ is any positive integer such that $\left(i, p_{j}\right)=1$ if $k_{j}>0$, and define $\left(i ; k_{1}, k_{2}, \cdots\right.$, $\left.k_{j}, \cdots\right)=\left(i ; k_{j}\right)$ to be the set of all rational numbers of the form $a i / b$, where $a$ is any integer and $b$ is an integer such that $b=\Pi_{p_{j}}^{\prime} p_{j}^{n_{j}}$ with $n_{j} \leq k_{j}$. Then each sequence determines a well-defined set of rational numbers. The symbol $\Pi^{\prime}$ designates a product over an arbitrary subset of the primes that satisfy whatever conditions are put on them; $\Pi$ designates a product over all primes that satisfy the given conditions.

2. Characterization of the rational groups. We show that the nontrivial subgroups of $R$ are exactly the subsets $\left(i ; k_{j}\right)$ defined in the introduction.

THEOREM 1. The set $\left(i ; k_{j}\right)$ is a subgroup of $R^{+}$, the additive group of rational numbers. We have $\left(i ; k_{j}\right)=\left(i^{\prime} ; k^{\prime}{ }_{j}\right)$ if and only if $i=i^{\prime}, k_{j}=k_{j}^{\prime}$ for all $j$.

Received October 11, 1950. Presented to the American Mathematical Society, June $17,1950$.

Pacific J. Math. 1 (1951), 169-177. 
Proof. If $a i / b \in\left(i ; k_{j}\right), c i / d \in\left(i ; k_{j}\right)$, then $b=\Pi_{p_{j}}^{\prime} p_{j}^{n_{j}}, d=\Pi_{p_{j}}^{\prime} p_{j}^{m_{j}}$, and $[b, d]=\Pi_{p_{j}}^{\prime} p_{j}^{s_{j}}$, where $s_{j}=\max \left(n_{j}, m_{j}\right) \leq k_{j}$. Writing $[b, d]=b b^{\prime}=d d^{\prime}$, we have

$$
\frac{a i}{b}-\frac{c i}{d}=\frac{b^{\prime} a i}{[b, d]}-\frac{d^{\prime} c i}{[b, d]}=\frac{\left(b^{\prime} a-d^{\prime} c\right) i}{[b, d]} \in\left(i ; k_{j}\right) .
$$

It is clear that different sequences determine different subgroups.

In the sequel we need the following properties of a subgroup $G \neq 0$ of $R^{+}$.

(1) Every $\zeta \in G$ has the form $\zeta=a i / b,(a i, b)=1$, where $i$ is the least positive integer in $G$.

For every $\zeta$ we have $\zeta=m / b$, where $(m, b)=1$; and if $i$ is the least positive integer in $G$, then $m=a i+r$ and $m-a i \in G$ imply $r=0$.

(2) If $a i / b \in G, i \in G$, and $(a, b)=1$, then $i / b \in G$.

For there exist integers $k, l$ such that $k a+l b=1$ and

$$
\frac{i}{b}=\frac{(k a+l b) i}{b}=\frac{k a i}{b}+l i \in G \text {. }
$$

(3) If $a i / b \in G$ where $i$ is the least positive integer in $G$, and $(a, b)=1$, then $(i, b)=1$.

By (2), $i / b \in G$; and if $(i, b) \neq 1, h / b^{\prime} \in G$ with $h<i$. Then $b^{\prime}\left(h / b^{\prime}\right)=$ $h \in G$.

We assume in the proof of the remaining properties that the elements of $G$ are written in the canonical form $a i / b$ with $(a i, b)=1$ and $i$ the least positive integer in $G$.

(4) If $a i / b c \in G$, then $i / b \in G$.

For $c a i / b c=a i / b \in G$ and $i / b \in G$ by (2).

(5) If $a i / b, c i / d \in G$, and if $(b, d)=1$, then $i / b d \in G$. For by (2) we have For by (2) we have

$$
\frac{i}{b d}=\frac{(k b+l d) i}{b d}=\frac{k i}{d}+\frac{l i}{b} \in G .
$$

THE OREM 2. If $G \neq 0$ is a subgroup of $R^{+}$, then there exists a sequence $\left(i ; k_{1}, k_{2}, \cdots, k_{j}, \cdots\right)$ such that $G=\left(i ; k_{j}\right)$.

Proof. By (1), every $\zeta \in G$ has the form $\zeta=a i / b,(a i, b)=1$, where $i$ is the 
least positive integer in $G$. We write all elements of $G$ in this form. If, for every $l$, there exist $a i / b \in G$ such that $p_{j}^{l} \mid b$, let $k_{j}=\infty$. If not, let $k_{j}=\max k$ such that $p_{j}^{k} \mid b$ for some $a i / b \in G$. Since $(a i, b)=1$, we have $\left(i, p_{j}\right)=1$ if $k_{j}>0$. By the definition of $i$ and $k_{j}, G$ is contained in $\left(i ; k_{j}\right)$. Now every element of $\left(i ; k_{j}\right)$ has the form $a i /\left(p_{1}^{n_{1}} \cdots p_{r}^{\left.n_{r}\right)}\right.$, where $n_{j} \leq k_{j}$ and $\left(a, p_{1}^{n_{1}} \cdots p_{r}^{n_{r}}\right)=1$. By (4) and the definition of $k_{j}, G$ contains every $i / p_{j}^{n_{j}}$ with $n_{j} \leq k_{j}$, and by (5), $G$ contains ai $/\left(p_{1}^{n_{1}} \cdots p_{r}^{n_{r}}\right)$. Hence $G=\left(i ; k_{j}\right)$.

3. Properties of the rational groups. In this section, properties of the rational groups are obtained as corollaries of the theorems of $\S 1$.

Corollary 1 . The group $\left(i ; k_{j}\right)$ is a subgroup of $\left(i^{\prime} ; k_{j}^{\prime}\right)$ if and only if $k_{j} \leq k_{j}^{\prime}$ and $i=m i^{\prime}$.

COROLLARY 2. The group $\left(i ; k_{j}\right)$ is cyclic if and only if $k_{j}<\infty$ for all $j$ and $k_{j}=0$ for almost all $j$.

Proof. If $\left(i ; k_{j}\right)$ is cyclic, it is generated by $a i / b$ with $(a i, b)=1$. Since every element of $\left(i ; k_{j}\right)$ has the form nai/b, we have $a=1$ and $b=\Pi_{k_{j}>0} p_{j}^{k_{j}}$. Conversely $\left(i ; k_{j}\right)$ contains $i / \Pi_{k_{j}>0} p_{j}^{k_{j}}$, and this element generates $\left(i ; k_{j}\right)$.

Corollary 3 . We have $\left(i ; k_{j}\right) \cong\left(i^{\prime} ; k_{j}^{\prime}\right)$ if and only if both $k_{j}=k_{j}^{\prime}$ for almost all $j$, and, whenever $k_{j} \neq k_{j}^{\prime}$, both are finite. Every isomorphism between $\left(i ; k_{j}\right)$ and $\left(i^{\prime} ; k_{j}^{\prime}\right)$ is given by

$$
\frac{a i}{b} \leftrightarrow \frac{m a i^{\prime}}{n b}
$$

where

$$
\begin{aligned}
& m=\left(\prod_{k_{j}=k_{j}^{\prime}=\infty}^{\prime} p_{j}^{a_{j}}\right)\left(\prod_{\substack{k_{j} \geq k_{j}^{\prime} \\
k_{j} \text { finite }}} p_{j}^{k_{j}-k_{j}^{\prime}}\right), \\
& n=\left(\prod_{k_{h}=k_{h}^{\prime}=\infty}^{\prime} p_{h}^{b_{h}}\right)\left(\prod_{\substack{k_{h}^{\prime} \geq k_{h} \\
k_{h}^{\prime} \text { finite }}} p_{h}^{k_{h}^{\prime}-k_{h}}\right) .
\end{aligned}
$$

Proof. If $\left(i ; k_{j}\right) \cong\left(i^{\prime} ; k^{\prime}\right)$, then $i \rightarrow m i^{\prime} / n$ with $\left(m i^{\prime}, n\right)=1$. If $\eta \longrightarrow i^{\prime}$, then $m \eta \longrightarrow m i^{\prime}$ and $n i \longrightarrow m i^{\prime}$, so that $m \eta=n i$, or $\eta=n i / m$. 
Hence $n i / m \longrightarrow i^{\prime}$. We write

$$
m=p_{\alpha_{1}}^{a_{1}} \cdots p_{\alpha_{r}}^{a_{r}}, \quad a_{l}>0, \quad n=p_{\beta_{1}^{1}}^{b_{1}} \cdots p_{\beta_{s}}^{b_{s}}, \quad b_{m}>0 ;
$$

then for $n_{j} \leq k_{j}$ we have

$$
\frac{i}{p_{j}^{n_{j}}} \rightarrow \frac{p_{\alpha_{1}}^{a_{1}} \cdots p_{\alpha_{r}}^{a_{r}} i^{\prime}}{p_{j}^{n_{j}} p_{\beta_{1}}^{b_{1}} \cdots p_{\beta_{s}}^{b_{s}}}
$$

while for $n_{j}^{\prime} \leq k_{j}^{\prime}$ we have

$$
\frac{p_{\beta_{1}}^{b_{1}} \cdots p_{\beta_{s}}^{b_{s}} i}{p_{j}^{n_{j}^{\prime}} p_{\alpha_{1}}^{a_{1}} \cdots p_{\alpha_{r}}^{a_{r}}} \rightarrow \frac{i^{\prime}}{p_{J}^{n_{j}^{\prime}}} .
$$

We have the following alternatives with consequences which follow from (3):

I.

$$
j=a_{l} \quad: n_{j}-k_{j}^{\prime} \leq a_{l} \leq k_{j}-n_{j}^{\prime}
$$

II.

$$
j=\beta_{m} \quad: \quad n_{j}^{\prime}-k_{j} \leq b_{m} \leq k_{j}^{\prime}-n_{j}
$$

III.

$$
\left.\begin{array}{l}
j \neq a_{l} \\
j \neq \beta_{m}
\end{array}\right\} \quad: \quad n_{j} \leq k_{j}^{\prime}, \quad n_{j}^{\prime} \leq k_{j}
$$

It follows that $k_{j}=\infty$ implies $k_{j}^{\prime}=\infty$ and conversely. With both $k_{j}$ and $k_{j}^{\prime}$ finite we may choose $n_{j}=k_{j}$ and $n_{j}^{\prime}=k_{j}^{\prime}$ and we have:

I.

$$
j=a_{l} \quad: a_{l}=k_{j}-k_{j}^{\prime}
$$

II.

$$
j=\beta_{m} \quad: \quad b_{m}=k_{j}^{\prime}-k_{j}
$$

III.

$$
\left.\begin{array}{l}
j \neq \alpha_{l} \\
j \neq \beta_{m}
\end{array}\right\} \quad: \quad k_{j}=k_{j}^{\prime}
$$

We have $k_{j}=k^{\prime}{ }_{j}$ if and only if $j \neq \alpha_{l}, j \neq \beta_{m}$. In particular, we have $k_{j}=k^{\prime}{ }_{j}$ for almost all $j$. If $k_{j}>k^{\prime}{ }_{j}$, then $j=\alpha_{l}$ and $a_{l}=k_{j}-k^{\prime}{ }_{j}$. If $k^{\prime}{ }_{j}>k_{j}$, then $j=\beta_{m}$ and $b_{m}=k_{j}^{\prime}-k_{j}$.

Now $i \longrightarrow m i^{\prime} / n$ implies $a i / b \longrightarrow a m i^{\prime} / b n$, so that the only isomorphisms between $\left(i ; k_{j}\right)$ and $\left(i^{\prime} ; k_{j}{ }_{j}\right)$ are those described in the corollary. Incidentally, we 
have derived necessary conditions for the relation $\left(i ; k_{j}\right) \cong\left(i^{\prime} ; k_{j}^{\prime}\right)$.

With the necessary conditions satisfied, we check that the given correspondence actually is an isomorphism. These conditions imply that the correspondence is single-valued with a single-valued inverse from $\left(i ; k_{j}\right)$ onto $\left(i^{\prime} ; k^{\prime}{ }_{j}\right)$. It is clear that addition is preserved.

COROLLARY 4. The group $\left(i ; k_{j}\right)$ admits only the identity automorphism if and only if $k_{j}$ is finite for all $j$.

Proof. If $k_{j}$ is finite for all $j$, we have by Corollary 3 , with $k_{j}=k_{j}^{\prime}$ for all $j$, that $m=n=1$. Conversely, if any $k_{j}=\infty$, then the correspondence of Corollary 3 gives us nontrivial automorphisms.

The multiplicative group of the field of rational numbers, $R_{.}^{\times}$, is a direct product of the infinite cyclic subgroups of $R^{\times}$generated by the prime numbers $p_{k}$ for all $k$. Such a subgroup consists of the elements $p_{k}, p_{k}^{2}, \cdots, 1,1 / p_{k}, 1 / p_{k}^{2}, \cdots$.

COROLLARY 5. The group of automorphisms of $\left(i ; k_{j}\right)$ is isomorphic to the direct product of all of the infinite cyclic subgroups of $R^{\times}$generated by those primes $p_{k}$ for which $k_{j}=\infty$.

Proof. By Corollary 3, there is a $(1-1)$ correspondence between the automorphisms of $\left(i ; k_{j}\right)$ and the rational numbers $M / N$ with $(M, N)=1$, where $M$ and $N$ are arbitrary products of those primes for which $k_{j}=\infty$. This correspondence clearly preserves multiplication and the set of all rationals $M / N$ has the stated structure as a group with respect to multiplication.

Corolla RY 6. For any two subgroups $\left(i ; k_{j}\right)$ and $\left(i^{\prime} ; k^{\prime}{ }_{j}\right)$ of $R^{+}$, the set $T$ consisting of all ordinary products of an element of $\left(i ; k_{j}\right)$ with an element of $\left(i^{\prime} ; k_{j}^{\prime}\right)$ is again a subgroup of $R^{+}$.

Proof. We have $T=\left(I ; K_{j}\right)$, where

$$
I=\frac{i i^{\prime}}{\Pi_{p_{j}} p_{j}^{s_{j}}}, \quad K_{j}=k_{j}+k_{j}^{\prime}-s_{j}
$$

with $s_{j}=\min \left(\alpha_{j}, k_{j}^{\prime}\right)+\min \left(\alpha_{j}^{\prime}, k_{j}\right)$, where $\alpha_{j}$ is the highest power of $p_{j}$ that divides $i$, and $\alpha_{j}^{\prime}$ the highest power of $p_{j}$ that divides $i^{\prime}$.

COR OLla RY 7. If $\left(i ; k_{j}\right) \geq\left(i^{i} ; k_{j}^{\prime}\right)$ and $p_{j}^{l_{j}}$ is the maximum power of $p_{j}$ such that $p_{j}^{l} l_{j}$ divides $i^{\prime} / i$, then the difference group $\left(i ; k_{j}\right)-\left(i^{\prime} ; k_{j}^{\prime}\right)$ is a direct sum 
of the groups $G_{j}$ where

(i) $G_{j}$ is the cyclic group,

$$
\left\{\left(i^{\prime} ; k_{J}^{\prime}\right)+\frac{i^{\prime}}{p_{j}^{k_{j}+l_{j}}}\right\},
$$

if $k_{j}$ is finite;

(ii) $G_{j}$ is the group of type $p^{\infty}$,

$$
p^{\infty}\left\{\left(i^{\prime} ; k_{j}^{\prime}\right)+\frac{i^{\prime}}{p_{j}^{k_{j}^{\prime}+1}}, \quad\left(i^{\prime} ; k_{j}^{\prime}\right)+\frac{i^{\prime}}{p_{j}^{k_{j}^{\prime}+2}}, \cdots\right\},
$$

if $k_{j}$ is infinite and $k_{j}^{\prime}$ is finite;

(iii) $G_{j}=\{0\}$ if $k_{j}=k_{j}^{\prime}=\infty$.

4. Rings which have a rational group as additive group. The distributive laws in any ring $S$ with $\left(i ; k_{j}\right)$ as additive group are used to determine all possible definitions of multiplication in $S$.

LemMA. If $S$ is a ring with additive group $\left(i ; k_{j}\right)$, then multiplication in $S$ is defined by

$$
\frac{a i}{b} \times \frac{c i}{d}=\frac{a c}{b d}(i \times i)
$$

Proof. We prove this by showing that

$$
b d\left(\frac{a i}{b} \times \frac{c i}{d}\right)=a c(i \times i) \text {. }
$$

We have

$$
\begin{array}{rlr}
a c(i \times i) & =a i \times c i & \text { (by the distributive laws in } S) \\
& =\left[b\left(\frac{a i}{b}\right)\right] \times\left[d\left(\frac{c i}{d}\right)\right] \\
& =\left[\begin{array}{cc}
\left.\frac{a i}{b}+\cdots+\frac{a i}{b}\right] \times\left[\frac{c i}{d}+\cdots+\frac{c i}{d \text { summands }}\right. \\
b
\end{array}\right]
\end{array}
$$


whence $a c(i \times i)$

$$
\begin{aligned}
& \left.=\left[\frac{a i}{b} \times \frac{c i}{d}\right]+\cdots+\left[\frac{a i}{b} \times \frac{c i}{d}\right] \quad \text { (by the distributive laws in } S\right) \\
& =(b d)\left[\frac{a i}{b} \times \frac{c i}{d}\right] .
\end{aligned}
$$

THЕОRЕM 3. If there is an infinite number of $k_{j}$ such that $0<k_{j}<\infty$, then the only ring $S$ with $\left(i ; k_{j}\right)$ as additive group is the null ring. If $0<k_{j}<\infty$ for only a finite number of $k_{j}$, then $S$ is a ring with additive group $\left(i ; k_{j}\right)$ if and only if multiplication in $S$ is defined by

$$
\frac{a i}{b} \times \frac{c i}{d}=\frac{a c A^{\prime}\left(\prod_{0<k_{j}<\infty} p_{j}^{k_{j}}\right) i}{b d \prod_{k_{j}=\infty}^{\prime} p_{j}^{n_{j}}},
$$

where $A^{\prime}$ and $n_{j}$ are arbitrary.

Proof. If $S$ is a ring with additive group $\left(i ; k_{j}\right)$, then $i \times i=A i / B \in\left(i ; k_{j}\right)$, where $(A i, B)=1, B=\Pi^{\prime} p_{j}^{n_{j}}, n_{j} \leq k_{j}$. By the lemma, we have

$$
\frac{a i}{b} \times \frac{c i}{d}=\frac{a c A i}{b d B}
$$

If $0<k_{r}<\infty$, this yields in particular

$$
\frac{i}{p_{r}^{k_{r}}} \times \frac{i}{p_{r}^{k_{r}}}=\frac{A i}{p_{r}^{2 k_{r} B}} .
$$

Therefore $\left(p_{r}, B\right)=1$, for otherwise we would have $2 k_{r}+n_{r} \leq k_{r}$, which is impossible. Hence, $B=\Pi^{\prime} p_{j}^{n_{j}}$ is a product of primes for which $k_{j}=\infty$, and it is necessary that $p_{r}^{k_{r}} \mid A$. If there is an infinite number of primes $p_{j}$ with $0<k_{j}<\infty$, then $A=0$ and $(a i / b) \times(c i / d)=0$. This proves the first statement in the theorem.

If $0<k_{j}<\infty$ for only a finite number of primes $p_{j}$, then

$$
A=A^{\prime} \prod_{0<k_{j}<\infty} p_{j}^{k_{j}}
$$


Together with what has been proved above, this gives

$$
\frac{a i}{b} \times \frac{c i}{d}=\frac{a c A^{\prime}\left(\prod_{0<k_{j}<\infty} p_{j}^{k_{j}}\right) i}{b d \prod_{k_{j}=\infty}^{\prime} p_{j}^{n_{j}}},
$$

where $A^{\prime}$ and $n_{j}>0$ are arbitrary integers.

Conversely, this definition of multiplication always makes $\left(i ; k_{j}\right)$ a ring. Closure with respect to $X$ is insured by providing $p_{j}^{k_{j}}$ in the numerator when $0<k_{j}<\infty$, and the associative and distributive laws are readily verified.

COROLLARY 1. The set $\left(i ; k_{j}\right)$ is a subring of $R$ if and only if there is no $k_{j}$ such that $0<k_{j}<\infty$.

Proof. Let $\left(i ; k_{j}\right)$ be a subring of $R$ and assume that for at least one $k_{j}$ we have $0<k_{j}<\infty$. If $0<k_{j}<\infty$ for infinitely many $k_{j}$, then $\left(i ; k_{j}\right)$ is not a subring of $R$, since by Theorem 3 it is the null ring. If $0<k_{j}<\infty$ for a finite number of $k_{j}$, then multiplication in any ring with $\left(i ; k_{j}\right)$ as additive group is given by the formula of the theorem. Hence this must reduce to ordinary multiplication for some choice of $A^{\prime}$ and $n_{j}$; that is,

$$
\frac{A^{\prime} \prod_{0<k_{j}<\infty} p_{J}^{k_{j}}}{\prod_{k_{j}=\infty}^{\prime} p_{j}^{n_{j}}}=i ; \quad A^{\prime} \prod_{\substack{0<k_{j}<\infty \\ n_{j}}} p_{j}^{k_{j}}=i \prod_{k_{j}=\infty}^{\prime} p_{j}^{n_{j}} .
$$

By hypothesis, at least one $p_{j}$ with $k_{j}>0$ appears in the left member of the above equality. Since no prime appears in both products, we have $p_{j} \mid i$. This contradicts $\left(i, p_{j}\right)=1$ for $k_{j}>0$.

Conversely, let every $k_{j}$ be either 0 or $\infty$. By the theorem, we have

$$
\frac{a i}{b} \times \frac{c i}{d}=\frac{a c A^{\prime} i}{b d \prod_{k_{J}=\infty}^{\prime} p_{J}^{n_{J}}},
$$

and we may select $A^{\prime}=i, \Pi_{k_{j}=\infty}^{\prime} p_{j}^{n_{j}=1}$, yielding ordinary multiplication.

Corolla $\mathrm{x}$ 2. If $\left(i ; k_{j}\right)$ is a subring of $R$, then $\left(i ; k_{j}\right)$ is a ring under the 
multiplication

$$
\frac{a i}{b} \times \frac{c i}{d}=\frac{a c}{b d}\left(\frac{e i}{f}\right)
$$

for arbitrary ei/f $\in\left(i ; k_{j}\right)$.

Proof. By Corollary 1, we have $k_{j}=0$ or $k_{j}=\infty$, so that every element of $\left(i ; k_{j}\right)$ has the form

$$
\frac{A^{\prime} i}{\prod_{k_{j}=\infty}^{\prime} p_{j}^{n_{j}}}
$$

and by the theorem these are just the multipliers which are used to define multiplication.

CoR OLLARY 3. If $S$ is a ring with additive group $\left(i ; k_{j}\right)$, then either $S$ is a null ring or $S$ is isomorphic to a subring of $R$.

Proof. If $S$ is not null, the correspondence

$$
\frac{a i}{b} \rightarrow \frac{a A}{b B}, \quad \text { where } \quad \frac{a i}{b} \times \frac{c i}{d}=\frac{a c A i}{b d B},
$$

is (1-1) from $S$ on a subset of $R$, and

$$
\begin{gathered}
\frac{a i}{b}+\frac{c i}{d}=\frac{(d a+b c) i}{b d} \rightarrow \frac{(d a+b c) A}{b d B}=\frac{a A}{b B}+\frac{c A}{d B}, \\
\frac{a i}{b} \times \frac{c i}{d}=\frac{a c A i}{b d B} \rightarrow \frac{a c A^{2}}{b d B^{2}}=\frac{a A}{b B} \frac{c A}{d B} .
\end{gathered}
$$

CoR OLLARY 4. All rings with additive group $R^{+}$are is omorphic to $R$. Proof. The correspondence of Corollary 3 clearly exhausts $R$.

\section{REFERENCE}

1. R. Baer, Abelian groups without elements of finite order, Duke Math. J. 3 (1937), 68-122. 



\title{
PACIFIC JOURNAL OF MATHEMATICS
}

\section{EDITORS}

\author{
Herbert BuSEMANN \\ R. M. RoBINSON \\ University of Southern California \\ University of California \\ Los Angeles 7, California \\ Berkeley 4, California \\ E. F. BEC KENBACH, Managing Editor \\ University of California \\ Los Angeles 24, California
}

\section{ASSOCIATE EDITORS}
R. P. DILWORTH
P. R. HALMOS
BØRGE JESSEN
J. J. STOKER
HERBERT FEDERER
HEINZ HOPF
PAUL LÉVY
MARSHALL HALL
R. D. JAMES
GEORGE PÓLYA
E. G. STRAUS
KÖSAKU YOSIDA

\section{SPONSORS}

UNIVERSITY OF BRITISH COLUMBIA

CALIFORNIA INSTITUTE OF TECHNOLOGY

UNIVERSITY OF CALIFORNIA, BERKELEY

UNIVERSITY OF CALIFORNIA, DAVIS

UNIVERSITY OF CALIFORNIA, LOS ANGELES

UNIVERSITY OF CALIFORNIA, SANTA BARBARA

OREGON STATE COLLEGE

UNIVERSITY OF OREGON
UNIVERSITY OF SOUTHERN CALIFORNIA

STANFORD UNIVERSITY

WASHINGTON STATE COLLEGE

UNIVERSITY OF WASHINGTON

AMERICAN MATHEMATICAL SOCIETY

NATIONAL BUREAU OF STANDARDS, INSTITUTE FOR NUMERIGAL ANALYSIS

Mathematical papers intended for publication in the Pacific Journal of Mathematics should be typewritten (double spaced), and the author should keep a complete copy. Manuscripts may be sent to any of the editors. All other communications to the editors should be addressed to the managing editor, E. F. Beckenbach, at the address given above.

Authors are entitled to receive 100 free reprints of their published papers and may obtain additional copies at cost.

The Pacific Journal of Mathematics is published quarterly, in March, June, September, and December. The price per volume (4 numbers) is $\$ 8.00$; single issues, $\$ 2.50$. Spécial price to individual faculty members of supporting institutions and to members of the American Mathematical Society: $\$ 4.00$ per volume; single issues, $\$ 1.25$.

Subscriptions, orders for back numbers, and changes of address should be sent to the publishers, University of California Press, Berkeley 4, California.

UNIVERSITY OF CALIFORNIA PRESS - BERKELEY AND LOS ANGELES 


\section{Pacific Journal of Mathematics}

\section{Vol. 1, No. $2 \quad$ December, 1951}

Tom M. (Mike) Apostol, On the Lerch zeta function ................. 161

Ross A. Beaumont and Herbert S. Zuckerman, A characterization of the subgroups of the additive rationals ....................... 169

Richard Bellman and Theodore Edward Harris, Recurrence times for the Ehrenfest model................................... 179

Stephen P.L. Diliberto and Ernst Gabor Straus, On the approximation of a function of several variables by the sum of functions of fewer

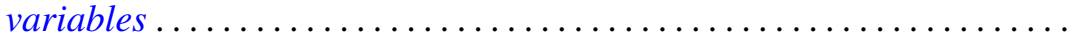

Isidore Isaac Hirschman, Jr. and D. V. Widder, Convolution transforms with complex kernels ................................ 211

Irving Kaplansky, A theorem on rings of operators .............. 227

W. Karush, An iterative method for finding characteristic vectors of a symmetric matrix............................... 233

Henry B. Mann, On the number of integers in the sum of two sets of positive integers ......................................... 249

William H. Mills, A theorem on the representation theory of Jordan

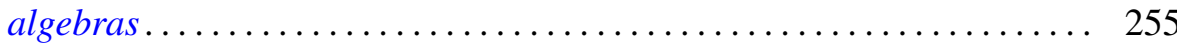

Tibor Radó, An approach to singular homology theory.............. 265

Otto Szász, On some trigonometric transforms ................... 291

James G. Wendel, On isometric isomorphism of group algebras ......... 305

George Milton Wing, On the $L^{p}$ theory of Hankel transforms ... 